\title{
CARACTERÍSTICAS DE GESTANTES ATENDIDAS EM CONSULTA DE ENFERMAGEM AMBULATORIAL DE PRÉ-NATAL: COMPARAÇÃO DE QUATRO DÉCADAS ${ }^{a}$
}

\author{
Ana Gabriela B. MARQUES , Suzana A. ZÁCHIAc, \\ Maria Luiza S. SCHMIDT ${ }^{\text {d }}$ Elizeth HELDT
}

\section{RESUMO}

O objetivo do trabalho foi identificar as características das gestantes atendidas em consulta de enfermagem no pré-natal realizado por enfermeira obstétrica em consulta ambulatorial, e compará-las, no período de 1972 a 2009. Os dados sociodemográficos e obstétricos foram coletados das fichas de gestantes, preenchidas durante a consulta de enfermagem. Um total de 1245 fichas foi analisado, sendo 208 (16,7\%) da década de 1970, 323 (25,9\%) de 1980,329 $(26,4 \%)$ de 1990 e 385 (30,0\%) de 2000. Encontrou-se diferença significativa entre as décadas anteriores com a de 2000, em relação ao número maior de: gestações de alto risco, número de consultas de enfermagem e de ecografias realizadas durante o pré-natal. Observa-se que as características das gestantes modificaram-se, ao longo do tempo, bem como o atendimento realizado por enfermeira obstétrica em consulta de enfermagem ambulatorial, mantendose vinculado às demandas das usuárias e às determinações legais.

Descritores: Cuidado pré-natal. Políticas públicas. Enfermagem obstétrica.

\section{RESUMEN}

El objetivo del trabajo fue identificar las características de las gestantes atendidas en consulta de enfermería prenatal, realizado por enfermera obstétrica en consulta de ambulatorio, y compararlas en el período de 1972 a 2009. Los datos sociodemográficos y obstétricos se recolectaron de las fichas de gestantes rellenadas durante la consulta de enfermería. Un total de 1245 fichas fueron analizadas, 208 (16,7\%) en la década de 1970, 323 (25,9\%) en la década de de 1980, 329 (26,4\%) en la década de 1990 y 385 (30,0\%) en la década del 2000. Se encontró diferencia significativa entre las décadas anteriores con la de 2000 con relación al número mayor de: gestaciones de alto riesgo, número de consultas de enfermería y de ecografías realizadas durante el prenatal. Se observa que las características de las gestantes se modificaron a lo largo del tiempo, así como la atención realizada por enfermería obstétrica en consulta de enfermería de ambulatorio, manteniéndose vinculado a las demandas de las usuarias y a las determinaciones legales.

Descriptores: Atención prenatal. Políticas públicas. Enfermería obstétrica.

Título: Características de las gestantes atendidas en consulta de enfermería en ambulatorio de prenatal: comparación de cuatro décadas.

\section{ABSTRACT}

The aim of the work was to identify characteristics of pregnant women cared for by an obstetric nurse in a visit to the prenatal outpatient nursing service and compare these over the period from 1972 to 2009. Sociodemographic and obstetric data were collected from the forms completed by the pregnant patients during their visit to the nursing service. A total of 1245 forms were analyzed, 208 (16.7\%) being from the 1970s, 323 (25.9\%) from the 1980s, 329 (26.4\%) from the 1990s, and 385 (30.0\%) from year 2000. A significant difference was found between the previous decades and year 2000 in relation to the greater number of high-risk pregnancies, number of nursing consultations and obstetric ultrasounds performed during the prenatal exam. The characteristics of pregnant women were observed to change over time, as well as the care provided by the obstetric nurse during visits to the outpatient nursing service, remaining associated with the demands of patients and legal resolutions.

Descriptors: Prenatal. Public policies. Obstetric nursing.

Title: Characteristics of pregnant women cared for in a visit to the prenatal oupatient nursing service: comparison of four decades.

a Estudo originário de um Trabalho de Conclusão de Curso da Escola de Enfermagem (EENF) da Universidade Federal do Rio Grande do Sul (UFRGS) e desenvolvido pelo Grupo de Pesquisa em Enfermagem Ambulatorial e em Atenção Básica (GPEAMAB), registrado no Diretório de Grupos de Pesquisa do Brasil - CNPq.

b Enfermeira graduada pela EENF/UFRGS, Porto Alegre, Rio Grande do Sul, Brasil.

c Enfermeira do Serviço de Enfermagem em Saúde Pública do Hospital de Clínicas de Porto Alegre (SESP-HCPA), Mestre em Ciências Médicas pela Faculdade de Medicina (FAMED) / UFRGS, Porto Alegre, Rio Grande do Sul, Brasil.

d Enfermeira do SESP - HCPA, Mestre em Enfermagem pela EENF/UFRGS, Porto Alegre, Rio Grande do Sul, Brasil.

e Professora Adjunta da EENF/UFRGS e dos Programas de Pós-Graduação em Enfermagem da EENF/UFRGS e de Pós-Graduação em Psiquiatria da FAMED/UFRGS. Doutora em Psiquiatria pela FAMED/UFRGS, Porto Alegre, Rio Grande do Sul, Brasil. 


\section{INTRODUÇÃO}

Há quatro décadas, o cuidado de enfermagem no pré-natal tem sido desenvolvido por enfermeiras obstétricas por meio da consulta de enfermagem no Serviço de Enfermagem em Saúde Pública (SESP) no ambulatório do Hospital de Clínicas de Porto Alegre $(\mathrm{HCPA})^{(1)}$.

Desde o início, em 1972, a consulta de enfermagem foi estruturada com base nas normas estabelecidas pelo Ministério da Saúde (MS), visando manter a integridade das condições de saúde materna e fetal ${ }^{(1)}$. O registro da consulta de enfermagem, desde então, vem sendo realizado no prontuário e, os dados obstétricos também estão sendo registrados na carteira da gestante, com o objetivo de garantir que as informações sobre a evolução da gestação sejam acessíveis aos diferentes profissionais que atendem a gestante. Para fornecer um rápido acesso dos principais dados da gestante, foram elaboradas as fichas obstétricas que são preenchidas na primeira consulta de enfermagem da gestante. A utilização das mesmas vem se mantendo ao longo do tempo, a fim de agilizar a tomada de decisões durante as consultas. Nos atendimentos subsequentes, os dados são atualizados até a realização de um último registro com os dados sobre o parto e sobre o recém-nascido ${ }^{(2)}$.

No transcorrer das quatro décadas, ocorreram mudanças significativas nas políticas públicas de saúde com relação à saúde da mulher. Na década de 1970, enfocavam a função procriativa, que eram traduzidas em cuidados com ênfase na visão da mulher como mãe. Em 1982, a OMS fortaleceu a evolução da assistência à mulher grávida, passando a considerá-la como um grupo prioritário nas políticas públicas. Em 1983, foi criado o Programa Nacional de Atenção Integral à Saúde da Mulher (PAISM), incorporando um novo enfoque nas políticas públicas, centrado no conceito de saúde integral, com ênfase na função educativa ${ }^{(3)}$.

Durante a XI Conferência Nacional de Saúde, realizada no ano de 2000, foi elaborado um documento que prevê: o acesso universal das mulheres grávidas para realização do pré-natal em serviços de qualidade; a implantação efetiva de comitês de mortalidade materna em todos os estados e municípios; os cuidados especiais e adequados à gravidez; a capacitação dos gestores e dos profissionais de saúde como condições indispensáveis para a melhoria da atenção à saúde da mulher. Nesse mesmo ano, foi instituído o Programa de Humanização no Pré-Natal e Nascimento, visando a redução das altas taxas de morbimortalidade materna e perinatal, a adoção de medidas para garantia do acesso, cobertura e qualidade do acompanhamento, bem como, da assistência ao parto, puerpério e neonatal e, a ampliação das ações até então adotadas ${ }^{(4)}$. É possível observar que o MS tem procurado, com essas medidas, estimular o desenvolvimento de práticas que possibilitem um olhar além das dimensões biológicas e que, de certa forma, reflitam no atendimento pré-natal ${ }^{(5)}$.

A metodologia da consulta de enfermagem também se modificou no decorrer desse período. Houve mudanças no processo de raciocínio clínico, de acordo com a Sistematização da Assistência de Enfermagem $(\mathrm{SAE})^{(6-7)}$, nos registros das informações que, atualmente, são em prontuário online e, sobretudo, na qualidade da assistência, considerando as políticas de humanização $o^{(5)}$.

O presente trabalho tem como objetivo a identificação das características das gestantes atendidas em consulta de enfermagem no pré-natal ambulatorial, realizado por enfermeira obstétrica ao longo de quatro décadas. Este resgate histórico mostra-se relevante para subsidiar o atendimento e a definição de novos programas, possibilitando uma reflexão sobre a atual metodologia das consultas, além de possíveis mudanças para a qualificação da assistência.

\section{MÉTODO}

A amostra do presente estudo foi constituída por informações obtidas de fichas obstétricas de gestantes atendidas por enfermeiras no Programa de Saúde da Mulher do SESP do HCPA, no período de 1972 a 2009. Os dados sociodemográficos que constam na ficha são: idade, escolaridade, estado civil, ocupação e procedência. Dentre as informações obstétricas estão: o número de consultas de enfermagem e de exames, o tipo de gestação, as gestações prévias e o tipo de parto. Mais de 4000 fichas foram separadas por décadas e sorteadas aleatoriamente. Para que fosse possível realizar as estimativas através do tempo, de forma que a variância fosse maximizada, o cálculo de tamanho amostral por década foi, no mínimo, de 200 fichas. As fichas com dados ilegíveis foram excluídas e substituídas através de um novo 
sorteio na década correspondente. Já as fichas incompletas sorteadas, fizeram parte da amostra pelo fato de significarem um dado relevante para comparação dos registros realizados nas consultas de enfermagem ao longo desse período.

A análise estatística foi realizada, utilizando-se o pacote estatístico Statistical Package for the Social Sciences (SPSS), versão 16.0, e o nível de significância adotado foram $\alpha=0,05$ e IC95\%. Os dados foram descritos, através da média e desvio padrão, conforme distribuição para variáveis contínuas. Para as variáveis categóricas, foi utilizado frequência e percentual. Para comparar as características sociodemográficas e obstétricas ao longo das décadas, foram utilizados os testes qui-quadrado para tendência linear e ANOVA ou o Teste Kruskal-Wallis. Para identificar as diferenças, foi utilizado o Teste de Dunn, Tukey e resíduos ajustados. A avaliação da distribuição normal foi realizada através do teste de Kolmogorov-Smirnov e a homogeneidade das variâncias através do teste de Levene.

O estudo foi aprovado pelo Comitê de Ética e Pesquisa do HCPA ( $n^{\circ}$ 100606) e as informações foram coletadas das fichas obstétricas, de maneira que os sujeitos da pesquisa não fossem identificados diretamente ou por meio de identificadores, conforme o termo de compromisso com a utilização de dados assinado pelas pesquisadoras.

\section{RESULTADOS}

A amostra total do estudo foi de 1245 fichas obstétricas, divididas nas quatro décadas. As características sociodemográficas das gestantes estão apresentadas na Tabela 1. Foi encontrada diferença significativa, quando comparada a faixa etária das gestantes entre as décadas, tendo mais adolescentes menores de 15 anos e mulheres com mais de 35 anos na década de 2000.

Na variável escolaridade, observam-se menos gestantes de nível superior na década de 2000. Também foram observadas diferenças significativas entre os locais de procedência das gestantes encaminhadas para a consulta de enfermagem obstétrica. Até a década de 1980, predominava mulheres oriundas de Porto Alegre, enquanto que, a partir de 2000, a maior frequência foi da região metropolitana e do interior do estado. As demais características sociodemográficas não apresentaram diferenças significativas entre as décadas (Tabela 1).
Em relação às características obstétricas, pode-se verificar um aumento significativo do número de gestações prévias e de gestações de alto risco na década de 2000, em comparação com as demais décadas (Tabela 2). O número de consultas de enfermagem no pré-natal teve aumento significativo na década de 2000, assim como o número de ecografias solicitadas, dos partos sem episiotomia e das cesáreas.

\section{DISCUSSÃO}

Os resultados encontrados mostram que as características das gestantes atendidas em consulta de enfermagem no pré-natal modificaram-se ao longo do tempo e essas mudanças podem estar associadas com as políticas vigentes em cada período. Assim, é relevante discutir os resultados desse estudo, fazendo um resgate cronológico das políticas públicas de saúde na assistência à saúde da mulher.

As políticas vigentes na década de 1970 para a assistência à mulher estavam em transformação com a criação do Programa de saúde materno-infantil, o qual enfocava ações voltadas ao pré-natal, ao parto e ao puerpério, tendo como critério a relação risco e classe social. Na ocasião, a prioridade era assistência ao mais pobre, porém, as ações ainda eram incipientes e a mulher era assistida de forma restrita, reducionista e fragmentada. Somente no final da década de 1970, os movimentos sociais articularam-se e pressionaram o governo a formular e a executar programas com estratégias voltadas à atenção materno-infantil. Logo, o interesse pelo tema saúde da mulher crescia no país não apenas nos espaços acadêmicos, mas também nos movimentos sociais organizados ${ }^{(8)}$.

Na década de 1980, o Programa de Assistência Integral a Saúde da Mulher (PAISM) foi implantado, sendo o pioneiro no cenário mundial ao propor o atendimento à saúde reprodutiva das mulheres no âmbito da atenção integral, não mais focada em ações isoladas de planejamento familiar. O cenário da atenção à mulher no Brasil começa a mudar, nesse período, com a distribuição de documentos técnicos para as secretarias estaduais as quais norteariam as chamadas "ações básicas de assistência integral a saúde da mulher", tornando, assim, o atendimento sistemático e baseado em conhecimentos científicos ${ }^{(3)}$. Isso se torna perceptível nos registros da ficha obstétrica, uma vez que, no final 
Tabela 1 - Comparação das características sociodemográficas das gestantes na consulta de enfermagem entre as quatro décadas. Porto Alegre, RS, 2012.

\begin{tabular}{|c|c|c|c|c|c|c|}
\hline \multirow{3}{*}{ Características } & \multirow{3}{*}{$\begin{array}{c}\text { Total } \\
n=1245\end{array}$} & \multicolumn{4}{|c|}{ Décadas } & \multirow{3}{*}{$\mathbf{p}$} \\
\hline & & 1970 & 1980 & 1990 & 2000 & \\
\hline & & $208(16,7 \%)$ & $323(25,9 \%)$ & $329(26,4 \%)$ & $385(30,9 \%)$ & \\
\hline Idade & $25,80 \pm 6,29$ & $26,38 \pm 4,78$ & $25,67 \pm 5,57$ & $25,39 \pm 6,80$ & $25,95 \pm 7,07$ & $0,365^{*}$ \\
\hline$\leq 15$ anos & $31(2,5)$ & --- & $3(0,9)-$ & $11(3,4)$ & $17(4,4)+$ & \\
\hline 16-35anos & $1106(89,6)$ & $195(94,2)+$ & $296(93,4)+$ & $288(88,1)$ & $327(85,2)-$ & $0,001^{* *}$ \\
\hline$>35$ anos & $98(7,9)$ & $12(5,8)$ & $18(5,7)$ & $28(8,6)$ & $40(10,4)+$ & \\
\hline
\end{tabular}

\section{Escolaridade}

\begin{tabular}{|c|c|c|c|c|c|c|}
\hline Fundamental & $233(45,2)$ & --- & --- & $89(42,6)$ & $144(47,1)$ & \\
\hline Médio & $221(42,9)$ & --- & --- & $84(40,2)$ & $137(44,8)$ & $0,008 * *$ \\
\hline Superior & $61(11,8)$ & --- & --- & $36(17,2)+$ & $25(8,2)-$ & \\
\hline \multicolumn{7}{|l|}{ Profissão } \\
\hline Do lar & $193(34,5)$ & --- & --- & $67(31)$ & $126(36,6)$ & \multirow{4}{*}{$0,562^{* *}$} \\
\hline Estudante & $82(14,6)$ & --- & ---- & $32(14,8)$ & $5 \mathrm{O}(14,5)$ & \\
\hline Empregada & $274(48,9)$ & --- & --- & $112(51,9)$ & $162(47,1)$ & \\
\hline Desempregada & $11(2)$ & --- & --- & $5(2,3)$ & $6(1,7)$ & \\
\hline \multicolumn{7}{|l|}{ Procedência } \\
\hline Porto Alegre & $837(69,3)$ & $185(88,9)+$ & $234(74,3)+$ & $223(69,7)$ & $195(53,4)-$ & \multirow{3}{*}{$<0,001^{* *}$} \\
\hline Grande P. Alegre & $329(27,2)$ & $22(10,6)-$ & $78(24,8)$ & $93(29,1)$ & $136(37,3)+$ & \\
\hline Interior & $42(3,5)$ & $1(0,5)-$ & $3(1)-$ & $4(1,3)-$ & $34(9,3)+$ & \\
\hline \multicolumn{7}{|l|}{ Estado Civil } \\
\hline Com companheiro & $582(93,1)$ & --- & ---- & $238(91,9)$ & $344(94)$ & \multirow{2}{*}{$0,308^{* *}$} \\
\hline Sem companheiro & $43(6,9)$ & --- & --- & $21(8,1)$ & $22(6)$ & \\
\hline
\end{tabular}

Nota: Variáveis contínuas estão apresentadas como média e desvio padrão e foram analisadas com ANOVA*. As variáveis categóricas estão apresentadas como frequência absoluta e relativa (\%) e foram analisadas com o teste Qui-quadrado** e pelos resíduos ajustados, sendo que + indica as categorias onde os valores observados foram significativamente maiores do que o esperado e - indica os que foram menores.

Fonte: Marques AGB, et al. Características de gestantes atendidas em consulta de enfermagem ambulatorial de pré-natal: comparação de quatro décadas. Rev Gaúcha Enferm, 2012.

dessa década, a ficha foi reformulada, passando a conter mais dados obstétricos, como, por exemplo, a idade gestacional, as ecografias obstétricas, a pressão arterial, a altura uterina, a circunferência abdominal e a do colo uterino.

$\mathrm{Na}$ década de 1990, as conquistas na área da saúde da mulher só progrediram. Nessa época, inicia um movimento pela redução de cesáreas desnecessárias. Em 1998, com a Portaria 2816, foi implementado um aumento de $160 \%$ na remuneração do parto normal, e instituído o pagamento de analgesia de parto, para coibir o abuso das cesarianas ${ }^{(9)}$. Nos resultados encontrados na pesquisa, é possível constatar que nas décadas de 1990 e 2000, o número de cesáreas foi significativamente maior comparado às outras décadas. Dessa forma, considerando que o HCPA é hospital de referência para gestação de risco $^{(10)}$, o aumento dos partos cesarianos pode estar relacionado ao maior número de gestantes de alto risco, que também cresceu significativamente nestas décadas, coerente com a política vigente. Em 2000, um estudo realizado no Hospital das Clínicas da Universidade de São Paulo mostrou que 74,75\% das mulheres estudadas apresentaram patologias associadas à gestação de risco ${ }^{(11)}$.

Na década de 2000, foi lançado o Programa de Humanização do Pré-natal e Nascimento (PHPN), pela Portaria 569, o qual previa o incentivo à assis- 
Tabela 2 - Comparação das características obstétricas e de atendimentos das gestantes entre as quatro décadas. Porto Alegre, RS, 2012.

\begin{tabular}{lcccccc}
\hline \multirow{2}{*}{ Características } & Total & \multicolumn{5}{c}{ Décadas } \\
\cline { 4 - 6 } & $\mathbf{n = 1 2 4 5}$ & $\mathbf{1 9 7 0}$ & $\mathbf{1 9 8 0}$ & $\mathbf{1 9 9 0}$ & $\mathbf{2 0 0 0}$ & $\mathbf{P}$ \\
\hline Prévias & & $\mathbf{2 0 8}(\mathbf{1 6 , 7 \%})$ & $\mathbf{3 2 3}(\mathbf{2 5 , 9 \%})$ & $\mathbf{3 2 9 ( 2 6 , 4 \% )}$ & $\mathbf{3 8 5 ( 3 0 , 9 \% )}$ & \\
Gestações & $2,02 \pm 1,35$ & $1,82 \pm 1,15^{\mathrm{a}}$ & $1,79 \pm 1,07^{\mathrm{a}}$ & $2,01 \pm 1,47^{\mathrm{a}}$ & $2,34 \pm 1,48^{\mathrm{b}}$ & $<0,001^{*}$ \\
Abortos & $0,30 \pm 0,66$ & $0,22 \pm 0,58^{\mathrm{a}}$ & $0,25 \pm 0-59^{\mathrm{ab}}$ & $0,34 \pm 0,72^{\mathrm{ab}}$ & $0,35 \pm 0,70^{\mathrm{b}}$ & $0,038^{*}$ \\
Parto Normal & $0,56 \pm 1,01$ & $0,49 \pm 0,90^{\mathrm{ab}}$ & $0,43 \pm 0,82^{\mathrm{a}}$ & $0,54 \pm 1,08^{\mathrm{ab}}$ & $0,72 \pm 1,11^{\mathrm{b}}$ & $0,001^{*}$ \\
Cesáreo & $0,15 \pm 0,46$ & $0,08 \pm 0,30^{\mathrm{a}}$ & $0,10 \pm 0,37^{\mathrm{a}}$ & $0,13 \pm 0,36^{\mathrm{a}}$ & $0,27 \pm 0,62^{\mathrm{b}}$ & $<0,001^{*}$ \\
\hline
\end{tabular}

Atual

Consulta de

Enfermagem

$1^{\circ}$ em idade gestacional

Total por gestação

$22,85 \pm 8,36$

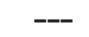

$19,25 \pm 6,45$

$23,42 \pm 8,37$

$22,51 \pm 8,36$

O,193**

Gestação de

$1,76 \pm 1,02$

$2,63 \pm 1,52^{\mathrm{a}}$

$3,11 \pm 1,59^{\mathrm{b}}$

$3,23 \pm 2,09^{b}$

$4,04 \pm 2,29^{\mathrm{C}}$

$<0,001 * *$

alto risco

$450(36,1)$

$43(20,7)^{\mathrm{a}}$

$100(31)^{b}$

$104(31,6)^{b}$

$203(52,7)^{\mathrm{c}}$

$<0,001^{* * * *}$

Total de Ecografias/

gestação

$3,35 \pm 2,01$

$0,23 \pm 0,46^{\mathrm{a}}$

$0.89 \pm 0,86^{\mathrm{b}}$

$2,02 \pm 1,14^{\mathrm{c}}$

$<0,001^{* *}$

Tipos de parto

Sem episiotomia

$\begin{array}{cc}107(16,3) & 1(3,8)- \\ 308(46,9) & 20(76,9)^{+} \\ 44(6,7) & --- \\ 192(29,9) & 5(19,9)\end{array}$

$38(16,1)$

$3(10,5)$

$45(25,7)^{+}$

$118(50)$

$111(50,5)$

$59(33,7)^{-}$

$<0,001^{*}$

Uso de fórceps

$20(8,5)$

$19(8,6)$

$5(2,9)^{-}$

Cesariana

$56(23,7)^{-}$

$65(29,5)$

$66(37,7)^{+}$

Nota: Variáveis contínuas estão apresentadas como média e desvio padrão e foram analisadas com os testes Kruskal-Wallis* e ANOVA*** As médias com letras diferentes foram estatisticamente significativas pelo teste de Dunn e pelo teste de Tukey $(\mathrm{p}<0,05)$. As variáveis categóricas estão apresentadas como frequência absoluta e relativa (\%) e foram analisadas com o teste Qui-quadrado**** e pelos resíduos ajustados, sendo que + indica as categorias onde os valores observados foram significativamente maiores do que o esperado e - indica os que foram menores. Fonte: Marques AGB, et al. Características de gestantes atendidas em consulta de enfermagem ambulatorial de pré-natal: comparação de quatro décadas. Rev Gaúcha Enferm, 2012.

tência ao pré-natal, estimulando os estados e municípios a realizarem o acompanhamento completo das gestantes. Esta Portaria institui a classificação de risco gestacional, garantindo o atendimento destas gestantes em unidades de referência ambulatorial ou hospitalar, prevendo um mínimo de seis consultas durante o pré-natal e, pelo menos, uma consulta de puerpério $^{(4)}$. Essas medidas acabaram por modificar o perfil das gestantes atendidas no HCPA, fato observado nos resultados encontrados. Pode-se constatar, através dos registros das fichas, uma modificação nas características demográficas, clínicas e no atendimento na década de 2000. Isto é, ocorreu um aumento da faixa etária de menores de 15 anos e maiores de 35 anos, no número de gestantes de alto risco, no número de ecografias realizadas, bem como, no número de gestantes oriundas do interior do estado e da região metropolitana. Ademais, o aumento relacionado às ecografias obstétricas tem se repetido em vários estudos nos últimos anos. No Canadá, por exemplo, houve uma elevação substancial no uso de ecografias no pré-natal na década de $90^{(12)}$. Em um hospital universitário de Rio Grande (RS), 99,1\% das puérperas haviam realizado pelo menos um exame de ultrassonografia durante o pré-natal, com uma média de 2,3 exames por paciente ${ }^{(13)}$.

O HCPA firma-se como hospital de referência para gestação de risco ${ }^{(10)}$, não tendo, exclusivamente, o critério da territorialidade prevista nos princí- 
pios do Sistema Único de Saúde. Logo, reafirma-se, o princípio de hierarquização, pela diferenciação das demandas mais complexas para as instituições hospitalares de nível terciário.

Em 2004, emergiu a proposta que prioriza a promoção da saúde, por meio da Política Nacional de Assistência Integral à Saúde da Mulher (PNAISM). Esta nova política tem como base a avaliação de políticas anteriores, buscando preencher as lacunas deixadas em relação ao climatério, infertilidade, reprodução assistida, doenças infecto-contagiosas, portadoras de doenças crônicas e às mulheres HIV positivo. Desse modo, dá ênfase aos direitos sexuais e reprodutivos da mulher e à melhoria da atenção obstétrica $^{(4)}$.

De fato, é perceptível o impacto da implantação das leis e das políticas públicas voltadas para a saúde da mulher que ocorreram na década de 1980, intensificaram-se ao longo da década de 1990, estendendo-se até os anos 2000. Contudo, as modificações na prática clínica ocorreram gradativamente, comprovando que, para a aplicação das leis, é necessária uma mudança de paradigma que exige firmeza e constância dos gestores, apesar de ser um processo lento. Um problema que influencia esse processo é que as mudanças preconizadas pelas políticas estão ocorrendo basicamente no sistema de saúde, enquanto o sistema de ensino continua preparando profissionais dentro do modelo intervencionista, considerado inadequado ${ }^{(14)}$.

A utilização de uma ficha que contenha as principais informações da gestação foi descrita no PHPN, ganhando o nome de ficha perinatal. Descrita como um instrumento de registro dos dados obtidos em cada consulta para uso dos profissionais de saúde da unidade que contenha os principais dados de acompanhamento da gestante, do parto, do recém-nascido e do puerpério ${ }^{(15)}$. Portanto, o uso da ficha obstétrica durante a consulta facilita o acesso rápido às principais informações sobre a gestante, agilizando, durante a consulta e até mesmo fora do horário agendado, à identificação de problemas para a tomada de decisões. Entretanto, numa consulta que tem duração de, em média, 30 minutos, o preenchimento dos mesmos dados em diversos locais (carteira da gestante, ficha obstétrica e prontuário) acaba por tornar a consulta burocrática, diminuindo assim, a precisão dos registros.
Dessa maneira, considerando que esse estudo foi uma análise de dados secundários, baseado em informações obtidas das fichas obstétricas, as conclusões devem ser observadas com cautela. Contudo, a relevância está por se tratar de um resgate histórico de uma modalidade de atenção preconizada à saúde materno-infantil. Cabe salientar ainda, que o desenvolvimento e a evolução do cuidado de enfermagem ocorreram em uma instituição de ensino, colaborando também para a formação de recursos humanos ${ }^{(16)}$, inserido em uma realidade social e política.

Finalmente, é importante reiterar a necessidade do adequado registro e a facilidade de acesso aos mesmos, para que a assistência prestada durante e após a gestação seja de qualidade, assegurando assim, os direitos da gestante.

\section{CONCLUSÕES}

Podemos concluir que as características das gestantes modificaram-se ao longo do tempo, bem como, o atendimento realizado por enfermeira obstétrica em consulta de enfermagem ambulatorial, vinculado às demandas das usuárias, às políticas de atenção à saúde da mulher e às determinações legais.

Salientamos que a enfermagem obstétrica ambulatorial faz parte da história institucional do HCPA e vem adaptando-se às determinações legais, às exigências embasadas pelo método científico e necessidades sociais.

\section{REFERÊNCIAS}

1 Santos BRL, Paskulin LMG, Crosseti MGO. Consulta de Enfermagem: sistematização do cuidado. In: Tasca AM, organizador. Cuidado ambulatorial: consulta de enfermagem e grupos. Rio de Janeiro: EPU; 2006. p. 21-28.

2 Rigol JL, Espírito Santo LC. Perfil das gestantes adolescentes atendidas em consulta de enfermagem. Rev Gaúcha Enferm. 2001; 22(2): 122-140.

3 Ministério da Saúde (BR). Política nacional de atenção integral à saúde da mulher: princípios e diretrizes [Internet]. Brasília (DF): Ministério da Saúde; 2004 [citado 2010 nov 16]. Disponível em: http:// conselho.saude.gov.b/politica_mulher.pdf.

4. Ministério da Saúde(BR). Programa de humanização no pré-natal e nascimento [Internet]. Brasília (DF): 
Ministério da Saúde, 2004 [citado 2010 out 27]. Disponível em: http://dtr2001.saude.gov.br/editora/ produtos/livros/genero/livros.htm.

5 Lima MG. Representações sociais das gestantes sobre a gravidez e a consulta de enfermagem no pré-natal. [dissertação]. Brasília (DF): Universidade de Brasília; 2006.

6 Mccloskey J, Bulecheck GM. Classificação das Intervenções de Enfermagem (NIC). $4^{\mathrm{a}}$ ed. Porto Alegre: Artmed; 2008.

7 North American Nursing Association, organizador. Diagnósticos de enfermagem da NANDA International: definições e classificação - (2009/2011). Porto Alegre: Artmed; 2009.

8 Neto ETS, Alves KCG, Zorz M, Lima RCD. Políticas de Saúde Materna no Brasil: os nexos com indicadores de saúde materno-infantil. Saúde Soc. 2008; 17(2):107-19.

9 Ministério da Saúde (BR), Agência Nacional de Vigilância Sanitária. Portaria GM/MS n.466, de 14 de junho de 2000 [Internet]. Brasília (DF); 2000 [citado 2011 mar 20]. Disponível em: http://www. anvisa.gov.br/legis/portarias/466_98.htm.

10 Ramos JGL, Costa SM, Stuczynski VJ, Brietzke E. Morte materna em hospital terciário do Rio Grande do Sul - Brasil: um estudo de 20 anos. Rev Bras Ginecol. Obstet. 2003; 25(6):431-36.

\footnotetext{
Endereço do autor / Dirección del autor / Author's address

Elizeth Heldt

Escola de Enfermagem - UFRGS, Departamento de Assistência e Orientação Profissional

Rua São Manoel, 963, sl. 218

90620-1 10, Porto Alegre, RS

E-mail:eliz.h@globo.com
}

11 Tase TH. Caracterização das mulheres atendidas em um hospital escola referência para gestação de alto risco. [dissertação]. São Paulo: Universidade de São Paulo; 2000.

12 You JJ, Alter DA, Stukel TA, McDonald SD, Laupacis A, Liu Y, et al. Proliferation of prenatal ultrasonography. CMAJ. 2010; 182(2): 143-151.

13 Gonçalves CV, Costa JSD, Duarte G, Marcolin AC, Velleca LCL, Garlet G, et al. Avaliação da frequência de realização do exame físico das mamas, dacolpocitologia cervical e da ultrassonografia obstétricadurante a assistência pré-natal: uma inversão de valores. Rev Assoc Med Bras 2009; 55(3): 290-5.

14 Rattner D. Humanização na atenção a nascimentos e partos: ponderações sobre políticas públicas. Interface. 2009 ; 13:759-68.

15 Ministério da Saúde (BR). Portaria GM/MS n.569, de 01 de junho de 2000: institui o Programa de Humanização no Pré-natal e Nascimento [Internet]. Brasília (DF); 2000 [citado 2010 nov 16]. Disponível em: http://dtr2001.saude.gov.br/sas/PORTARIAS/ PORT2000/GM/GM-569.htm.

16 Marques AG. Características das gestantes atendidas em consulta de enfermagem ambulatorial de pré-natal: comparação de quatro décadas [monografia]. Porto Alegre: Escola de Enfermagem, Universidade Federal do Rio Grande do Sul; 2011.

Recebido em: 10.11 .2012

Aprovado em: 04.12.2012 